Objectives: We aim in our 3 years observational study to evaluate the predictive role of ILC2 in SSc patients.

Methods: We conducted an observational retrospective study on 52 patients with SSc fulfilling the 2013 ACR/EULAR classification criteria. Yearly clinical, laboratory and investigational data according to EUSTAR recommendations were collected. Blood samples collected between 15.09.2014 and 15.01.2015 were analyzed by flow cytometry and ILC2 counts were measured. The predictive value of ILC2 during a 3-year follow-up was analyzed using SPSS 21.0.

Results: 52 patients were included in the study, $78 \%$ female, $63 \%$ limited cutaneous SSc with a mean follow-up time of $2.85 \pm 1.28$ years. At baseline we have shown that circulating ILC2s are significantly increased compared to gender and age-matched healthy controls. Increased numbers of ILC2s significantly correlated with worsening of mRSS calculated by five point increase in $\mathrm{mRSS}$ or $25 \%$ increase from baseline $(p<0.001 ; 95 \% \mathrm{Cl} 1.39-3.26$ ). ILC2 counts also correlated with $5 \%$ decrease of diffusion capacity of carbon monoxide (DLCO) during the follow-up time ( $p<0.0001 ; 95 \% \mathrm{Cl} 1.83-3.49$ ). Worsening of forced vital capacity (FVC) assessed as $5 \%$ decrease over 2 years was also significantly correlated with an increased number of ILC2s $(p<0.0001 ; 95 \% \mathrm{Cl} 1.27-3.04)$. In contrast, we did not find any correlation regarding increase in pulmonary arterial pressure assessed by echocardiography. Although new appearance of digital ulcers could not be predicted by ILC2 counts, increased numbers of ILC2s were correlated with digital ulcers at follow-up.

Conclusion: Here, we provide first evidence for a role of ILC2s as potential prognostic marker of disease progression in SSc.

Disclosure of Interests: Alina Soare: None declared, Stefanie Weber: None declared, Georg Schett Speakers bureau: AbbVie, BMS, Celgene, Janssen, Eli Lilly, Novartis, Roche and UCB, Jörg Distler Grant/research support from: Boehringer Ingelheim, Consultant of: Boehringer Ingelheim, Paid instructor for: Boehringer Ingelheim, Speakers bureau: Boehringer Ingelheim, Andreas Ramming Grant/research support from: Pfizer, Novartis, Consultant of: Boehringer Ingelheim, Novartis, Gilead, Pfizer, Speakers bureau: Boehringer Ingelheim, Roche, Janssen

DOI: 10.1136/annrheumdis-2020-eular.6322

\section{SAT0343 NUTRITIONAL STATUS OF SYSTEMIC SCLEROSIS PATIENTS: A PILOT STUDY}

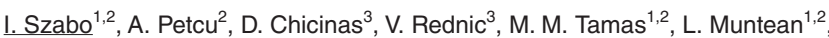
I. Filipescu ${ }^{1,2}$, S. P. Simon ${ }^{1,2}$, C. Pamfil ${ }^{2}$, S. Rednic ${ }^{1,2} .{ }^{1}$ County Emergency Clinical Hospital Cluj-Napoca, Rheumatology, Cluj-Napoca, Romania; ${ }^{2}$ University of Medicine an Pharmacy "Iuliu Hatieganu"Cluj-Napoca, ClujNapoca, Romania; ${ }^{3}$ Regional Institute of Gastroenterology and Hepatology "Octavian Fodor"Cluj-Napoca, Cluj-Napoca, Romania

Background: Gastrointestinal involvement (GI) in systemic sclerosis (SSc) is one of the major disease burdens. Its consequences on the nutritional status of SSc patients and their quality of life is poorly evaluated during routine check-ups. Since malnutrition is an important cause of morbidity and mortality, addressing this issue seems necessary.

Objectives: The aim of this study was to evaluate the risk of malnutrition in SSc patients and to identify potential associations between the risk of malnutrition and clinical features or laboratory parameters.

Methods: All patients aged $>18$ years old with a definite diagnosis of SSc according to the 2013 ACR/EULAR classification criteria from the EUSTAR Center 16 and ERN ReCONNET cohort of the County Emergency Clinical Hospital Cluj-Napoca were included in the study. Patients with localized scleroderma, scleroderma sine scleroderma, overlap syndromes and mixed connective tissue disease were excluded. Clinical and laboratory data was collected from the EUSTAR database and medical charts. A telephone survey was conducted and patients were interviewed using the Malnutrition Universal Screening Tool (MUST) questionnaire.

Results: 75 patients were eligible for the study. Female to male ratio was 10:1 with an almost equal distribution among limited (57\%) and diffuse (43\%) SSc subtypes. The most prevalent autoantibodies were anti-TOPO-I and anti-centromere. Gl symptoms were reported in $48.6 \%$ patients out of which $86 \%$ SSc patients underwent further evaluation by upper GI endoscopy. Abnormal endoscopic findings, such as esophagitis, Barret esophagus and gastritis were identified in $80 \%$ patients. Most patients had a low risk of malnutrition (93\%) with only a minority carrying a medium $(6 \%)$ or high $(1 \%)$ risk. No significant association was demonstrated between MUST score and the extend of cutaneous involvement (limited SSc versus diffuse SSc; $p=0.39$ ), presence of $\mathrm{Gl}$ symptoms $(p=0.35)$, presence of abnormal endoscopic findings $(p=0.45)$ or presence of anemia $(p=0.83)$
Conclusion: The majority of SSc patients from this cohort exhibited a low risk of malnutrition. These results are contradictory to previous literature reports. A possible explanation is that the MUST score is a dynamic screening too and therefore interviewing patients with a stable disease (outpatient care) versus patients with active disease (inpatient care) might lead to different results Another limitation of this study is the small number of patients included. This is a pilot study. We aim to further extend the study population to the other EUSTAR cohorts and to prospectively evaluate these patients in an inpatient care setting.

\section{References:}

[1] Dupont $\mathrm{R}$ et al. Impact of micronutrient deficiency \& malnutrition in systemic sclerosis: Cohort study and literature review. Autoimmun Rev. 2018 Nov;17(11):1081-1089;

[2] Caimmi C et al. Malnutrition and sarcopenia in a large cohort of patients with systemic sclerosis. Clin Rheumatol. 2018 Apr;37(4):987-997;

[3] Türk i et al. Malnutrition, associated clinical factors, and depression in systemic sclerosis: a cross-sectional study. Clin Rheumatol. 2020 Jan;39(1):57-67.

Disclosure of Interests: None declared

DOI: 10.1136/annrheumdis-2020-eular.6459

SAT0344 LIMITED JOINT MOBILITY OF HAND IN SYSTEMIC
SCLEROSIS PATIENTS BY USING "PRAYER" AND
"TABLE TOP" SIGNS

S. Uslu ${ }^{1}$, S. Gulle ${ }^{2}$, A. Koken Avsar ${ }^{2}$, A. Karakas² ${ }^{2}$ S. B. Kocaer ${ }^{2}$, T. Yüce İnel ${ }^{2}$, Y. Erez ${ }^{2}$, G. Can ${ }^{2}$, I. Sari ${ }^{2}$, F. Onen ${ }^{2}$, M. Birlik ${ }^{2} .{ }^{1}$ Ömer Halisdemir University Bor Physical Medicine and Rehabilitation, Training and Research Hospital, Department of Rheumatology, Niğde, Turkey; ${ }^{2}$ Dokuz Eylul University Faculty of Medicine, Department of Rheumatology, izmir, Turkey

Background: Limited joint mobility (LJM) is a musculoskeletal disorder caused by flexion contractures of hand is a common complication in systemic sclerosis (SSc) patients. The distal parts of the upper limb (hands and fingers) is the most involved site in SSc.

Objectives: In this study, we aimed to evaluate LJM in SSc patients and to determine the relationship between the clinical features of the disease.

Methods: A total of 113 patients ( $>18$ years old) diagnosed with diffuse cutaneous systemic sclerosis (DcSSc) and limited cutaneous systemic sclerosis (LcSSc) and 104 healthy controls were included in this study. LJM was evaluated with "prayer sign" and "table top sign" tests. LJM staging was done by Rosenbloom classification method(1, 2). LJM (+) and LJM (-) patients were compared in terms of demographic findings (gender, age and duration of disease), laboratory results (ESR, CRP, ANA, anti-topoisomerase I and anti-centromere) and modified Rodnan Skin Score (mRSS) results.

Results: In our study, a total of 113 patients diagnosed with SSc and 104 healthy controls with similar age and gender distribution were included. While LJM $(+)$ was detected in $75(66.4 \%)($ LcSSc $=38$, DcSSc $=37)$ of the patients diagnosed with SSc, LJM (mild) (+) was detected in $3(2.8 \%)$ of the control group. One of these people had right 2nd DIF joint contracture due to osteoarthritis, and 1 patient was found to have simple contractures due to minor hand injury previously (Table 1). A statistically significant difference was observed in between LcSSc and DcSSc patients according to the presence of LJM ( $<<0.001$ ) (Table 2). There was a moderate positivity relationship between LJM and mRSS (LcSSc: $r=0.449$ ve $p<0.001$, DcSSc: $r=0.565$ ve $p<0.001$ ) (Figure 1).

Table 1. Comparison of demographic findings between SSc and Control group

\begin{tabular}{|c|c|c|c|}
\hline & SSc Group ( $n=113)$ & Control Group ( $n=104)$ & $p$ value \\
\hline Age, year & $57.02 \pm 11.58$ & $58.47 \pm 11.26$ & 0.061 \\
\hline Gender (F / M) & $98(86.7) / 15(13.3)$ & 65 (62.5) / 39 (37.5) & 0.064 \\
\hline CRP (mg/L) & $5.45 \pm 5.39$ & $2.14 \pm 1.12$ & $<0.001$ \\
\hline ESR (mm/hr) & $25.19 \pm 18.9$ & $14.46 \pm 10.09$ & 0.024 \\
\hline \multicolumn{4}{|l|}{ Smoking, n (\%) } \\
\hline Smoker & 89 (78.8) & $70(67.3)$ & 0.464 \\
\hline Non-Smoker & 24 (21.2) & 34 (32.7) & \\
\hline \multicolumn{4}{|l|}{ LJM (Absent / Present) } \\
\hline Present & $75(66.4)$ & $3(2.8)$ & $<0.001$ \\
\hline Absent & $38(33.6)$ & $101(97.2)$ & \\
\hline Rosenbloom classification & $\operatorname{LcSSc}(n=71)(\%)$ & DcSSc $(n=42)(\%)$ & Total $(n=113)(\%)$ \\
\hline Normal & 46.5 & 11.9 & 33.6 \\
\hline Mild & 22.5 & 14.3 & 19.5 \\
\hline Moderate & 23.9 & 33.3 & 27.4 \\
\hline Severe & 7.1 & 40.5 & 19.5 \\
\hline
\end{tabular}


Table 2. Comparison of demographic and clinical findings LJM(-) and $\operatorname{LJM}(+)$ in SSc

\begin{tabular}{lccc}
\hline & LJM (-) (n=38) & LJM (+) (n=75) & p value \\
\hline Age, year & $54.16 \pm 11.82$ & $58.47 \pm 11.26$ & 0.061 \\
SSc Type & $\mathrm{n}(\%)$ & $\mathrm{n}(\%)$ & \\
LcSSc, n (\%) & $33(56.8)$ & $38(50.7)$ & $<0.001$ \\
DcSSc, n (\%) & $5(13.2)$ & $37(49.3)$ & \\
Gender, F/M (\%) & $37(97.3) / 1(2.7)$ & $61(81.3) / 14(18.7)$ & $\mathbf{0 . 0 1 8}$ \\
Raynaud's (symptom duration), month & $148(44-456)$ & $150(35-588)$ & 0.990 \\
Non-raynaud (symptom duration), month & $108(28-458)$ & $138(38-447)$ & 0.132 \\
mRSS, median & $2(0-14)$ & $8(0-36)$ & $<0.001$ \\
CRP (mg/L) & $4.21 \pm 4.48$ & $6.08 \pm 5.71$ & 0.069 \\
ESR (mm/hr) & $19.74 \pm 10$ & $27.95 \pm 21.6$ & 0.270 \\
Renal crisis, $\mathbf{n}(\%)$ & $1(2.6)$ & $4(5.3)$ & 0.662 \\
PAH, $\mathbf{n}(\%)$ & $8(21.1)$ & $14(18.7)$ & 0.762 \\
ANA positivity, $\mathbf{n}(\%)$ & $36(94.7)$ & $70(93.3)$ & 1 \\
Anti-centromere positivity, $\mathbf{n}$ (\%) & $18(47.4)$ & $19(25.3)$ & $\mathbf{0 . 0 1}$ \\
Anti-topoisomerase-1, $\mathbf{n}(\%)$ & $8(21)$ & $34(45.3)$ & $\mathbf{0 . 0 1}$ \\
Smoking, $\mathbf{n}(\%)$ & $\mathrm{n}(\%)$ & $\mathrm{n}(\%)$ & \\
Non-smoker & $30(78.9)$ & $59(78.7)$ & 0.970 \\
Smoker & $8(21.1)$ & $16(21.3)$ & \\
\hline
\end{tabular}

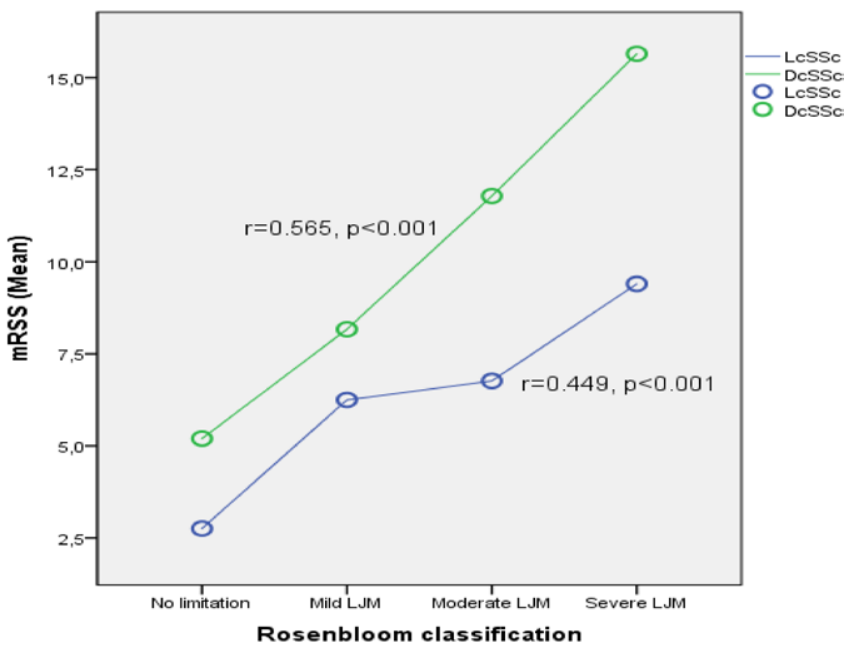

Figure 1.

Conclusion: In our study, it was found that LJM staging positively correlated with mRSS and DcSSc patients had more severe LJM findings than LcSSc. We conclude that "prayer sign" and "table top sign" tests used in hand evaluation in SSc patients have similar clinical results with mRSS and can be easily performed in daily practice in about 3 minutes.

References:

[1] Rosenbloom AL. Limitation of finger joint mobility in diabetes mellitus. The Journal of diabetic complications 1989; 3: 77-87.

[2] Nashel J, Steen V. Scleroderma mimics. Current rheumatology reports 2012; 14: 39-46.

Disclosure of Interests: None declared

DOI: 10.1136/annrheumdis-2020-eular.4494

\section{SAT0345 IS THERE A DIFFERENCE BETWEEN THE SEXES IN THE RATE OF PROGRESSION OF SYSTEMIC SCLEROSIS-ASSOCIATED ILD (SSC-ILD)? DATA FROM THE SENSCIS TRIAL}

E. Volkmann ${ }^{1}$, S. Vettori ${ }^{2}$, J. Varga ${ }^{3}$, A. Herrick ${ }^{4}$, M. Cutolo ${ }^{5}$, A. Cordeiro ${ }^{6}$, V. F. Azevedo ${ }^{7}$, S. Johnson ${ }^{8}$, C. Stock ${ }^{9}$, M. Gahlemann ${ }^{10}$, L. Moros ${ }^{11}$, M. Alves12, M. Mayes ${ }^{13} .{ }^{1}$ Department of Medicine, Division of Rheumatology, University of California, David Geffen School of Medicine, Los Angeles, California, United States of America; ${ }^{2}$ Università degli Studi della Campania “Luigi Vanvitelli", Naples, Italy; ${ }^{3}$ Northwestern Scleroderma, Feinberg School of Medicine, Chicago, Illinois, United States of America; ${ }^{4}$ Centre for Musculoskeletal Research, The University of Manchester, Salford Royal NHS Foundation Trust, Manchester, United Kingdom; ${ }^{5}$ Research Laboratory and Academic Unit of Clinical Rheumatology, Department of Internal Medicine, University of Genova, IRCCS San Martino Polyclinic Hospital, Genova, Italy; ${ }^{6}$ Department of Rheumatology, Hospital Garcia de
Orta, Almada, Portugal; ${ }^{7}$ Hospital de Clínicas, Federal University of Paraná, Curitiba, Paraná, Brazil; ' ${ }^{8}$ Division of Rheumatology, Department of Medicine, Toronto Western Hospital, Mount Sinai Hospital; Institute of Health Policy, Management and Evaluation, University of Toronto, Toronto, Ontario, Canada;

${ }^{9}$ Boehringer Ingelheim Pharma GmbH \& Co. KG, Biberach, Germany;

${ }^{10}$ Boehringer Ingelheim (Schweiz) GmbH, Basel, Switzerland; ${ }^{11}$ Boehringer Ingelheim International GmbH, Ingelheim, Germany; ${ }^{11}$ Boehringer Ingelheim International $\mathrm{GmbH}$, Ingelheim, Germany; ${ }^{13}$ Division of Rheumatology and Clinical Immunogenetics, University of Texas McGovern Medical School, Houston, Texas, United States of America

Background: Previous studies suggested that male sex may be associated with a greater rate of decline in FVC in patients with SSc-ILD. In the SENSCIS trial, nintedanib reduced the rate of FVC decline over 52 weeks vs placebo.

Objectives: Analyse the rate of decline in FVC and the efficacy and safety of nintedanib in the SENSCIS trial in subgroups by sex.

Methods: Patients with SSc-ILD with first non-Raynaud symptom $<7$ years before screening and $\geq 10 \%$ fibrosis of the lungs on HRCT were randomised to nintedanib or placebo. We analysed the rate of decline in FVC (mL/year) and adverse events over 52 weeks in male and female patients.

Results: Of 576 patients, $433(75.2 \%)$ were female. Compared with males the female subgroup included a smaller proportion of White patients $(64.7 \%$ vs $74.8 \%$ ), a smaller proportion on mycophenolate at baseline (46.9\% vs $53.1 \%$ ) a greater proportion of ATA positive patients $(63.3 \%$ vs $53.1 \%$ ), and had a lower mean weight at baseline $(66.6 \mathrm{vs} 79.1 \mathrm{~kg})$. FVC \% predicted $(72.8 \%$ vs $71.7 \%$ ) and mRSS (11.2 vs 10.8$)$ were similar in females and males. The adjusted annual rate of decline in FVC in the placebo group was numerically greater in male than female patients (-126.8 [SE 29.0] vs -82.0 [16.2] mL/year). The estimated effect of nintedanib vs placebo on reducing the rate of decline in FVC was numerically more pronounced in males than females (difference: $58.6[95 \% \mathrm{Cl}-18.0,135.1]$ vs $34.6[-9.3,78.4] \mathrm{mL} /$ year $)$, but the interaction $\mathrm{p}$-value did not indicate heterogeneity in the treatment effect between subgroups $(p=0.59)$. Among nintedanib-treated patients, diarrhoea was reported in similar proportions of females and males $(74.7 \%$ vs $79.1 \%)$; nausea, vomiting and liver test abnormalities were reported in greater proportions of females vs males (35.3\% vs $19.4 \%, 28.1 \%$ vs $13.4 \%$, and $15.4 \%$ vs $9.0 \%$ ), while serious adverse events were more frequent in males $(32.8 \%$ vs $21.3 \%)$. In the nintedanib and placebo groups, respectively, adverse events leading to treatment discontinuation were reported in $16.7 \%$ and $8.5 \%$ of females and $13.4 \%$ and $9.2 \%$ of males.

Conclusion: In the SENSCIS trial in patients with SSc-ILD, the annual rate of decline in FVC in the placebo group was numerically greater in male than female patients. The rate of FVC decline was lower with nintedanib than placebo both in males and females. The safety profile of nintedanib was similar between males and females.

Figure. Rate of decline in FVC (mL/year) over 52 weeks in subgroups by sex in the SENSCIS trial

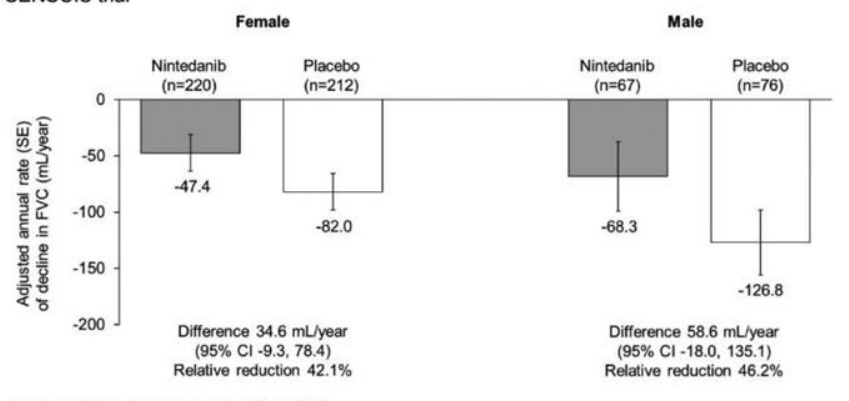

Disclosure of Interests: Elizabeth Volkmann Grant/research support from: Forbius, Corbus Pharmaceuticals, Consultant of: Boehringer Ingelheim, Forbius, Speakers bureau: Boehringer Ingelheim, Serena Vettori Consultant of: Boehringer Ingelheim, John Varga Grant/research support from: John Varga is awaiting grants from Boehringer Ingelheim and has received grants from Bristol-Myers Squibb, Pfizer, Takeda, and TeneoBio, Consultant of: John Varga has acted as a consultant for Boehringer Ingelheim, Bristol-Myers Squibb, Emerald Health, and TeneoBio, Ariane Herrick: None declared, Maurizio Cutolo Grant/ research support from: Bristol-Myers Squibb, Actelion, Celgene, Consultant of: Bristol-Myers Squibb, Speakers bureau: Sigma-Alpha, Ana Cordeiro Consultant of: Ana Cordeiro has acted as a consultant for Roche, Speakers bureau: Ana Cordeiro has received speaker fees from Boehringer Ingelheim, Lilly, and Vitoria, Valderilio F Azevedo Grant/research support from: Abbvie, Janssen, 